\title{
Phase Transitions and Low-temperature Structure of Lithium Manganese Oxide Spinel
}

\author{
Masao Yonemura, ${ }^{1,2}$ Takashi Kamiyama, ${ }^{3}$ Yoji Kawamoto ${ }^{2}$ and Ryoji Kanno ${ }^{1 *}$ \\ ${ }^{1}$ Department of Electronic Chemistry, Interdisciplinary Graduate School of Science and Engineering, \\ Tokyo Institute of Technology, Yokohama 226-8502, Japan \\ ${ }^{2}$ Department of Chemistry, Faculty of Science, Kobe University, Kobe 657-8501, Japan \\ ${ }^{3}$ Neutron Science laboratory, Institute of Materials Structure Science, High Energy Accelerator Research Organization, \\ Tsukuba 305-0801, Japan
}

Low-temperature structures of the lithium manganese spinels were determined using TOF neutron Rietveld analysis. The spinels $\mathrm{LiMn}_{2} \mathrm{O}_{4-\delta}$ with different $\delta$ values, $(0.016,0.040$, and 0.132$)$ showed the cubic-orthorhombic phase transitions, and the lattice distortion decreased with decreasing $\delta$. Although no anomaly corresponding to the cubic-orthorhombic phase transition was observed in the DSC curve for the spinel with $\delta \sim 0.016$, the transition was observed by the structure analysis, which is consistent with the broad $C p$ anomaly at $250 \mathrm{~K}$. The cubic-orthorhombic phase transition is closely correlated to the existence of the vacancy. The charge disproportionation into trivalent and tetravalent state proceeds gradually with decreasing temperature, and the extent of the disproportionation is dependent on the vacancy. Based on the structure analysis, the phase transitions in the spinel are discussed.

(Received February 4, 2004; Accepted April 15, 2004)

Keywords: lithium manganese spinel, phase transition, charge-ordering

\section{Introduction}

Lithium manganese oxide spinels are a promising candidate as cathodes in rechargeable lithium batteries because of their low cost and higher voltages than the layered rocksalt cathodes, $\mathrm{LiMO}_{2}(M=\mathrm{Co}, \mathrm{Ni}) .{ }^{1)}$ Cathodic properties of the lithium manganese spinels were influenced by synthesis conditions. ${ }^{2)}$ Although many studies have been tried to clarify the cathodic properties and to optimize the synthesis conditions, the dominant factors that determine their characteristics have remained obscure.

Another important aspect of the spinel is the phase transition associated with the Jahn-Teller trivalent manganese ion. Lattice distortion in the spinel is caused by the cooperative interaction of the Jahn-Teller $\mathrm{Mn}^{3+}$ ion on the octahedral site. Three types of phases related to co-operative Jahn-Teller ordering were reported; (i) the tetragonal phase synthesized at high-temperatures and quenched to liq $\mathrm{N}_{2}$, (ii) the tetragonal phase appeared during lithium intercalation from $\mathrm{LiMn}_{2} \mathrm{O}_{4}$, and (iii) the low-temperature phase appeared around room temperature. The tetragonal phase (i) observed at high-temperature was stabilized at room temperature by quenching from $920^{\circ} \mathrm{C}$. $^{3)}$ Its structure has a cooperative ordered arrangement of trivalent manganese ions. ${ }^{4,5)}$ The tetragonal phase (ii) appeared during the lithium intercalation has been studied in detail to clarify the charge-discharge characteristics of the $3 \mathrm{~V}$ region. The ordered arrangement of the elongated axis of the $\mathrm{MnO}_{6}$ octahedra was similar to the tetragonal $\mathrm{Mn}_{3} \mathrm{O}_{4}$ spinel. These types of ordering in the tetragonal phases (i) and (ii) are well known to the spinels with Jahn-Teller ions. Low-temperature phase (iii) was first reported by Yamada et al. ${ }^{6,7)}$ to be a tetragonal phase. The transition proceeds gradually from the high-temperature cubic phase to the low-temperature tetragonal phase through

*Corresponding author, E-mail: kanno@echem.titech.ac.jp two-phase region with decreasing temperature. Later, the low-temperature structure was indicated to be orthorhombic with superlattice reflections, ${ }^{8)}$ and the superstructure was solved by the $3 a \times 3 a \times 1 a$ lattice. ${ }^{9)}$ However, tetragonal symmetry was indicated again for the low-temperature phase, ${ }^{10)}$ and more recently, the mixed two-phase region was again indicated by synchrotron X-ray diffraction study. ${ }^{11)}$

Considerable confusion in understanding the spinel is partly due to the sample dependence of their physical properties. The transition behavior changed with the compositions which are dependent on synthesis procedures of each research groups. Significant discrepancy in understanding the spinel from the standpoint of solid-state chemistry is the presence of oxygen vacancy. The oxygen vacancy was indicated first by the thermogravimetry measurements. ${ }^{3,12,13)}$ Two structure models were proposed for the "oxygen vacancy" phase: vacancy at the oxygen site corresponding to the formula $\mathrm{LiMn}_{2} \mathrm{O}_{4-\delta}$, and excess cations at the interstitial site with the formula, $\mathrm{Li}_{1+x} \mathrm{Mn}_{2+y} \mathrm{O}_{4}$. These models were, in fact, claimed by experimental results; the oxygen deficient spinel $\mathrm{LiMn}_{2} \mathrm{O}_{4-\delta}$ with vacancy clustering based on the NMR data, ${ }^{13)}$ oxygen vacancy indicated by chemical analysis, ${ }^{14)}$ and interstitial cations at the $16 c$ octahedral site in $F d \overline{3} m$ space group determined by density measurement. ${ }^{15)}$

Recently, we studied the structures of the spinels synthesized at temperatures over a range of $750 \leq t \leq 900^{\circ} \mathrm{C}$ with $\mathrm{Li} / \mathrm{Mn}$ ratios between 0.5 and 0.55 and various starting materials. ${ }^{20)}$ The neutron Rietveld analysis of these samples indicated that the oxygen vacancy exits at the oxygen $32 e$ site, and the spinels with nearly stoichiometric composition was difficult to obtain. The system was divided into three categories: oxygen deficient spinels, $\mathrm{LiMn}_{2} \mathrm{O}_{4-\delta}$, lithium substituted spinels, $\mathrm{Li}_{1+x} \mathrm{Mn}_{2-x} \mathrm{O}_{4-\delta}$, and the stoichiometric spinel, $\mathrm{LiMn}_{2} \mathrm{O}_{4}$. The ideal composition of $\mathrm{Li}_{1} \mathrm{Mn}_{2} \mathrm{O}_{4}$, 
however, could not be obtained. Oxygen vacancy in the spinel affects their electrochemical and phase transition properties. The magnetic measurements also indicated several anomalies corresponding to the transition around room temperature, long-range magnetic ordering due to antiferromagnetic interaction near $50 \mathrm{~K}$, and short-range magnetic ordering (spin-glass-like behavior) around $10 \mathrm{~K}$. These transitions shift systematically with the amount of vacancy. ${ }^{19)}$

Based on our systematic characterization of the lithium manganese oxides spinel, re-examination of the chargeordering phase transition is necessary. In the present study, we determined their structures at low-temperatures. Neutron diffraction is expected to be a powerful means to clarify structural details, because of the difference in the coherent scattering lengths of $\mathrm{Li}, \mathrm{Mn}$ and $\mathrm{O}$ atoms $\left(b_{\mathrm{c}}(\mathrm{Li})=-1.900\right.$, $\left.b_{\mathrm{c}}(\mathrm{Mn})=-3.730, b_{\mathrm{c}}(\mathrm{O})=5.803 \mathrm{fm}\right)$. We confirmed the orthorhombic structure for the low-temperature phase, and the orthorhombic distortion decreased with decreasing oxygen vacancy, $\delta$. The relationship between the composition, structure, phase transitions is discussed.

\section{Experimental}

Three different samples were synthesized and used for low-temperature structure analysis. Table 1 summarizes the synthesis condition and room temperature structure parameters for the samples used in the present study. The synthesis procedure of these samples are as follows.

Sample A: The lithium manganese spinel was prepared by heating appropriate molar ratios of $\mathrm{Li}_{2} \mathrm{CO}_{3}$ and $\mathrm{Mn}_{2} \mathrm{O}_{3}$ $\left(\mathrm{Li}_{2} \mathrm{CO}_{3}\right.$ : Nacalai tesque, $>99.9 \%$ purity; $\mathrm{Mn}_{2} \mathrm{O}_{3}$ : High Purity Chemicals Laboratory Co., Ltd., $>99.9 \%$ purity). They were mixed, pelleted, and then heated at $900^{\circ} \mathrm{C}$ in an oxygen atmosphere and a cooling rate of $100^{\circ} \mathrm{C} / \mathrm{h}$. No impurity phase was observed. In the oxygen $32 e$ site, a small amount of vacancy was observed with a value of occupation parameter, $g=0.9666(16)$ at $325 \mathrm{~K}$. The composition determined by the structure analysis was $\mathrm{LiMn}_{2} \mathrm{O}_{3.868}$.

Sample B: The spinels close to the stoichiometric composition were synthesized from starting materials of the manganese oxide obtained by thermal decomposition of manganese oxalate at $400^{\circ} \mathrm{C}$ (Manganese oxide: Toda Kogyo Co., Ltd.). The appropriate molar ratio of $\mathrm{LiOH} \cdot \mathrm{H}_{2} \mathrm{O}$ ( $\mathrm{LiOH} \cdot \mathrm{H}_{2} \mathrm{O}$ : Nacalai tesque) and the manganese oxide were

Table 1 Rietveld refinement results for samples mesured by neutron diffraction experiments.

\begin{tabular}{|c|c|c|c|c|c|c|c|c|c|c|c|}
\hline \multirow[b]{2}{*}{$\begin{array}{c}\text { Sample } \\
\text { label }\end{array}$} & \multirow{2}{*}{\multicolumn{2}{|c|}{ Composition }} & \multirow[b]{2}{*}{$g(\mathrm{O})$} & \multirow{2}{*}{$\begin{array}{l}\text { Mixing } \\
\mathrm{Li} / \mathrm{Mn} \\
\text { ration }\end{array}$} & \multirow{2}{*}{$\begin{array}{l}\text { Lattiec pa- } \\
\text { rameters } \\
a / \mathrm{nm}\end{array}$} & \multirow[b]{2}{*}{$\begin{array}{l}\text { Starting } \\
\text { materials }\end{array}$} & \multicolumn{2}{|c|}{ Synthesis condition } & \multirow{2}{*}{$\begin{array}{l}\text { Cooling rate } \\
t /{ }^{\circ} \mathrm{Ch}^{-1}\end{array}$} & \multirow[b]{2}{*}{$\begin{array}{l}\text { Impurty } \\
\text { phase }\end{array}$} & \multirow{2}{*}{$\begin{array}{l}\text { Measurement } \\
\text { temperature } \\
T / \mathrm{K} \\
\end{array}$} \\
\hline & & & & & & & $\begin{array}{l}\text { Temperature } \\
t /{ }^{\circ} \mathrm{C}\end{array}$ & Atomsphare & & & \\
\hline A & $\mathrm{LiMn}_{2} \mathrm{O}_{4-\delta}$ & $\delta=0.132$ & $0.9666(16)$ & 0.5 & $0.824609(14)$ & $\mathrm{Li}_{2} \mathrm{CO}_{3}, \mathrm{Mn}_{2} \mathrm{O}_{3}$ & 900 & $\mathrm{O}_{2}$ & 100 & no & 325 \\
\hline B & $\mathrm{LiMn}_{2} \mathrm{O}_{4-\delta}$ & $\delta=0.040$ & $0.990(7)$ & 0.5 & $0.823803(8)$ & $\mathrm{LiOH}, \mathrm{MnO}_{x}$ & $470(1) / 750$ & Air $/ \mathrm{O}_{2}$ & 60 & no & 300 \\
\hline $\mathrm{C}$ & $\mathrm{LiMn}_{2} \mathrm{O}_{4-\delta}$ & $\delta=0.016$ & $0.996(7)$ & 0.5 & $0.823785(13)$ & $\mathrm{LiOH}, \mathrm{MnO}_{x}$ & $470(3) / 750$ & Air $/ \mathrm{O}_{2}$ & 60 & no & 300 \\
\hline
\end{tabular}
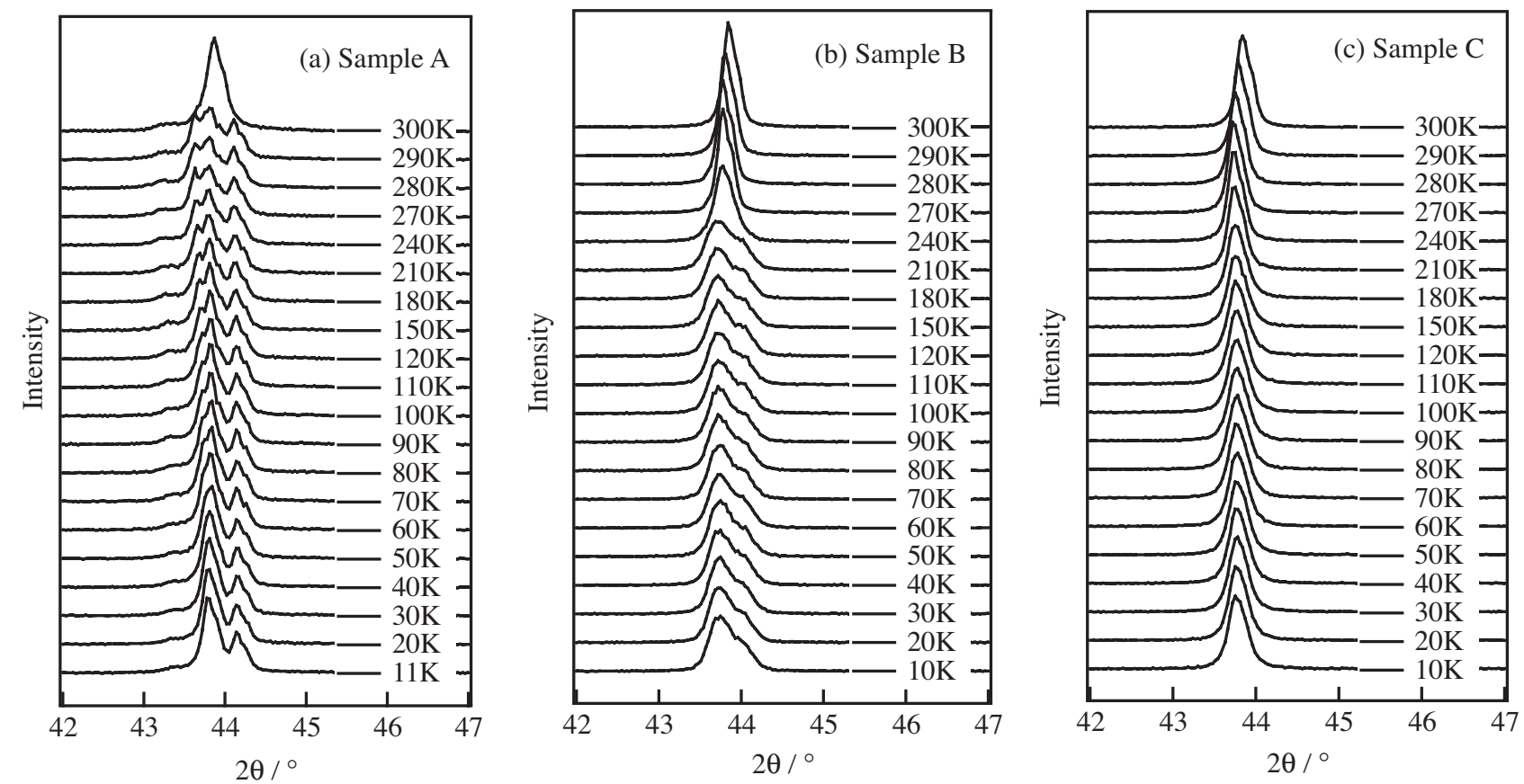

Fig. 1 (a) X-ray diffraction patterns of $\mathrm{LiMn}_{2} \mathrm{O}_{3.868}$ (sample A) between 10 and $300 \mathrm{~K}$. (b) X-ray diffraction patterns of $\mathrm{LiMn}_{2} \mathrm{O}_{3.960}$ (sample B) between 10 and $300 \mathrm{~K}$. (c) X-ray diffraction patterns of $\mathrm{LiMn}_{2} \mathrm{O}_{3.984}$ (sample C) between 10 and $300 \mathrm{~K}$. 

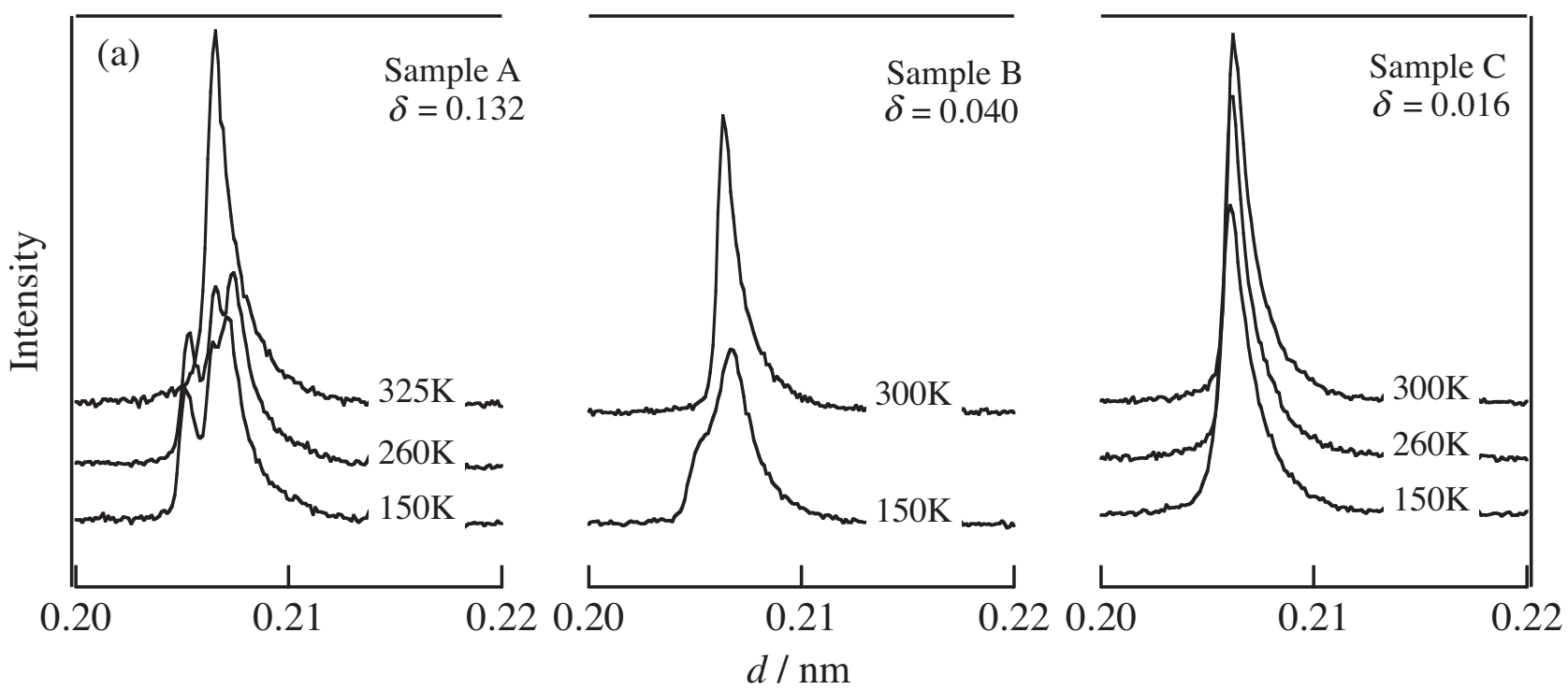

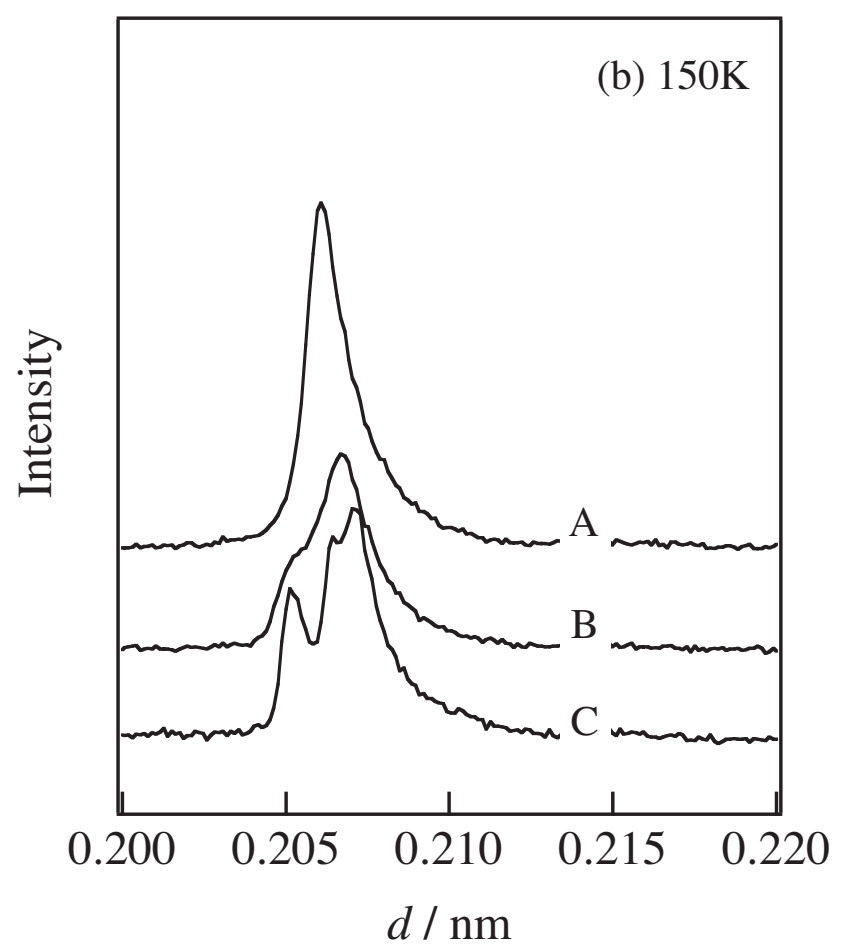

mixed with a $\mathrm{Li} / \mathrm{Mn}$ ratio of $1 / 2$, pelletized, and heated at $470^{\circ} \mathrm{C}$ for $6 \mathrm{~h}$ in air. The samples were then ground with pestle and mortar, and made pellets again. After the preheating process, these pellets were heated at $750^{\circ} \mathrm{C}$ for $24 \mathrm{~h}$ with a heating and cooling rate of $60^{\circ} \mathrm{C} \cdot \mathrm{h}^{-1}$. The neutron diffraction measurements indicated that the occupation parameter at the oxygen $32 e$ site was $0.990(7)$ at $300 \mathrm{~K}$. The composition determined was $\mathrm{LiMn}_{2} \mathrm{O}_{3.960}$.

Sample C: The sample C was synthesized by the same starting materials as the sample $\mathrm{B}$. The starting materials were mixed with a $\mathrm{Li} / \mathrm{Mn}$ ratio of $1 / 2$, pelletized, and heated at $470^{\circ} \mathrm{C}$ for $6 \mathrm{~h}$ in air. The samples were then ground with pestle and mortar, and made pellets again. The pre-heating process was repeated by 3 times. After the pre-heating process, these pellets were heated at $750^{\circ} \mathrm{C}$ for $24 \mathrm{~h}$ with a
Fig. 2 Neutron diffraction $(400)_{c}$ peaks at low temperatures for the samples A, B, and C (a). Peak splittings of $(400)_{c}$ reflection for the samples $\mathrm{A}, \mathrm{B}$, and $\mathrm{C}$ at $150 \mathrm{~K}$ (b). heating and cooling rate of $60^{\circ} \mathrm{C} \cdot \mathrm{h}^{-1}$. The room temperature structure analysis indicated that the amount of oxygen at the $32 e$ oxygen site was $0.998(6)$ at $300 \mathrm{~K}$. The composition determined was $\mathrm{LiMn}_{2} \mathrm{O}_{3.984}$.

$\mathrm{X}$-Ray diffraction patterns of the powdered samples were obtained with an X-ray diffractometer (Rigaku RAD-C, $12 \mathrm{~kW}$ ) with $\mathrm{CuK} \alpha$ radiation. The diffraction data were collected at each $0.02^{\circ}$ step width over a $2 \theta$ range from 10 to $100^{\circ}$. The low-temperature X-ray diffraction patterns were taken in the temperature range between 10 and $300 \mathrm{~K}$. Differential scanning calorimetry (DSC) was measured by a TAS-200 (Rigaku) between 150 and $360 \mathrm{~K}$ at a heating and cooling rate of $10 \mathrm{~K} / \mathrm{min}$. Neutron diffraction data for the spinels were taken between 5 and $325 \mathrm{~K}$ on a time-of-flight (TOF) neutron powder diffractometer, VEGA, at the KENS 


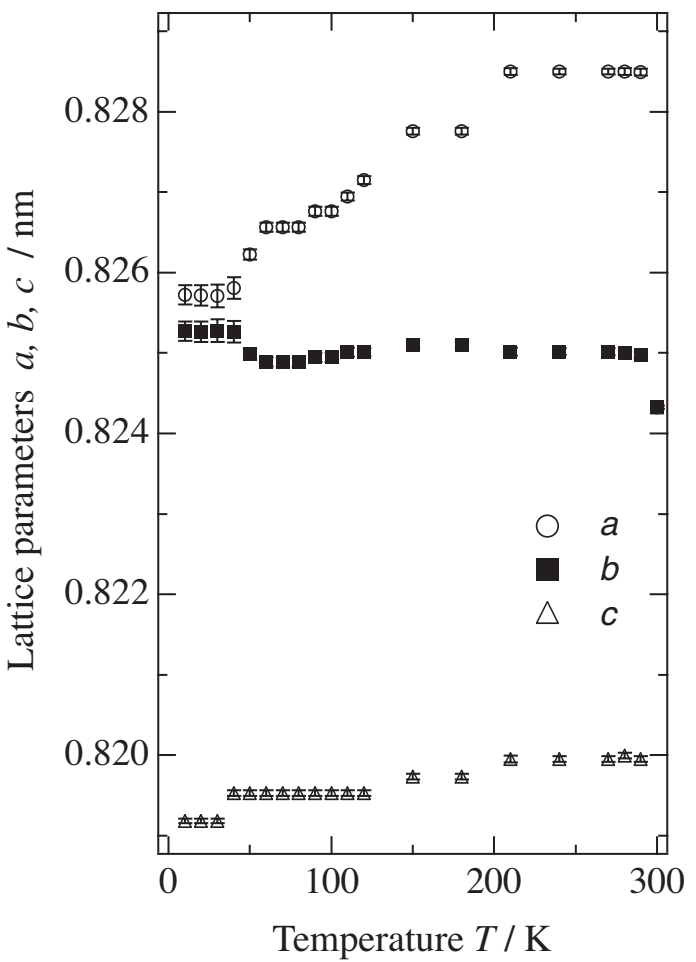

Fig. 3 Temperature dependence of lattice parameters for $\mathrm{LiMn}_{2} \mathrm{O}_{3.868}$ (sample A) between 10 and $300 \mathrm{~K}$. The data were obtained by X-ray diffraction measurements.

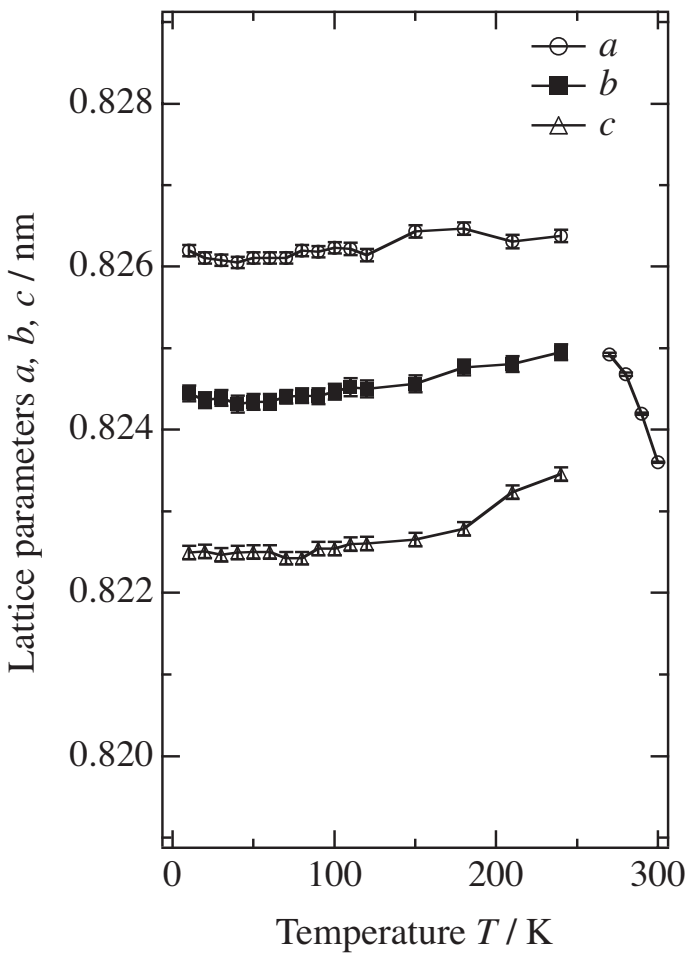

Fig. 4 Temperature dependence of lattice parameters for $\mathrm{LiMn}_{2} \mathrm{O}_{3.984}$ (sample C) between 10 and $300 \mathrm{~K}$. The data were obtained by X-ray diffraction measurements.

Table 2 Neutron Rietveld refinement results at $150 \mathrm{~K}$ for $\mathrm{LiMn}_{2} \mathrm{O}_{4-\delta}(\delta=0.132)$ (sample A).

\begin{tabular}{|c|c|c|c|c|c|c|}
\hline Atom & Site & $g$ & $x$ & $y$ & $z$ & $U / \mathrm{nm}^{2}$ \\
\hline Li1 & $32 h$ & 1 & $0.289(14)$ & $0.2869(8)$ & $0.127(3)$ & $0.0061(8)$ \\
\hline $\mathrm{Li} 2$ & $16 e$ & 1 & $0.293(17)$ & $5 / 8$ & $1 / 8$ & $=\operatorname{Li1}(B)$ \\
\hline $\mathrm{Li} 4$ & $16 f$ & 1 & $3 / 8$ & $0.547(1)$ & $3 / 8$ & $=\operatorname{Li1}(B)$ \\
\hline Mn1 & $16 d$ & 1 & $1 / 4$ & $1 / 4$ & $1 / 2$ & $0.016(15)$ \\
\hline $\mathrm{Mn} 2$ & $32 h$ & 1 & $0.2511(3)$ & $0.3337(5)$ & $0.7529(16)$ & $0.0024(7)$ \\
\hline Mn3 & $32 h$ & 1 & $0.3311(5)$ & $0.2435(3)$ & $0.7511(17)$ & $0.0036(8)$ \\
\hline Mn4 & $32 h$ & 1 & $0.3360(4)$ & $0.3273(3)$ & $0.4969(14)$ & $0.0010(8)$ \\
\hline Mn5 & $32 h$ & 1 & $0.3304(4)$ & $0.5848(4)$ & $0.7491(18)$ & $0.0029(7)$ \\
\hline $\mathrm{O} 1$ & $32 h$ & 1 & $0.2561(3)$ & $0.2585(2)$ & $0.7560(7)$ & $0.0100(10)$ \\
\hline $\mathrm{O} 2$ & $32 h$ & 1 & $0.2565(2)$ & $0.3279(2)$ & $0.5170(6)$ & $0.0056(7)$ \\
\hline $\mathrm{O} 3$ & $32 h$ & 1 & $0.2479(2)$ & $0.5897(2)$ & $0.7332(7)$ & $0.0061(9)$ \\
\hline $\mathrm{O} 4$ & $32 h$ & 1 & $0.3267(2)$ & $0.2452(2)$ & $0.5237(6)$ & $0.0022(7)$ \\
\hline O6 & $32 h$ & 1 & $0.3252(2)$ & $0.5871(2)$ & $0.5137(6)$ & $0.0064(8)$ \\
\hline $\mathrm{O} 7$ & $32 h$ & $0.78(1)$ & $0.3372(3)$ & $0.3334(3)$ & $0.2698(7)$ & $0.0035(8)$ \\
\hline O8 & $32 h$ & 1 & $0.3392(2)$ & $0.50965(12)$ & $0.7601(6)$ & $0.0024(7)$ \\
\hline O9 & $32 h$ & 1 & $0.3372(3)$ & $0.5844(2)$ & $0.9872(7)$ & $0.014(1)$ \\
\hline
\end{tabular}

Space group $F d d d, a=2.47449(4) \mathrm{nm}, b=2.48303(5) \mathrm{nm}, c=0.819607(17) \mathrm{nm}, R_{\mathrm{wp}}=6.21, R_{\mathrm{p}}=5.88, S=R_{\mathrm{wp}} / R_{\mathrm{e}}=1.0560, R_{\mathrm{I}}=1.59, R_{\mathrm{F}}=2.00$.

pulsed spallation neutron source at the National Laboratory for High Energy Accelerator (KEK). The specimen of (ca. $5 \mathrm{~g}$ ) was contained in a cylindrical vanadium cell of dimensions $5 \mathrm{~mm}$ in radius, $55 \mathrm{~mm}$ in height, and $200 \mu \mathrm{m}$ in thickness. The structural parameters were refined with RIETAN-2001T. ${ }^{16)}$

\section{Results and Discussion}

The structural changes with temperature were studied by $\mathrm{X}$-ray diffraction and neutron diffraction measurements. Figure 1 shows the X-ray diffraction patterns for the samples $\mathrm{A}, \mathrm{B}$, and $\mathrm{C}$, recorded from $300 \mathrm{~K}$ to $10 \mathrm{~K}$ on cooling. Figure 2 
Table 3 Neutron Rietveld refinement results at $200 \mathrm{~K}$ for $\operatorname{LiMn}_{2} \mathrm{O}_{4-\delta}(\delta=0.132)$ (sample A).

\begin{tabular}{|c|c|c|c|c|c|c|}
\hline Atom & Site & $g$ & $x$ & $y$ & $z$ & $U / \mathrm{nm}^{2}$ \\
\hline Li1 & $32 h$ & 1 & $0.2905(14)$ & $0.2884(11)$ & $0.123(4)$ & $0.0075(8)$ \\
\hline Li2 & $16 e$ & 1 & $0.2901(17)$ & $5 / 8$ & $1 / 8$ & $=\operatorname{Li1}(U)$ \\
\hline $\mathrm{Li} 3$ & $8 a$ & 1 & $3 / 8$ & $3 / 8$ & $7 / 8$ & $=\operatorname{Li1}(U)$ \\
\hline $\mathrm{Li} 4$ & $16 f$ & 1 & $3 / 8$ & $0.5452(16)$ & $3 / 8$ & $=\operatorname{Li1}(U)$ \\
\hline Mn1 & $16 d$ & 1 & $1 / 4$ & $1 / 4$ & $1 / 2$ & $0.0016(13)$ \\
\hline $\mathrm{Mn} 2$ & $32 h$ & 1 & $0.2513(3)$ & $0.3329(5)$ & $0.7561(15)$ & $0.0018(8)$ \\
\hline Mn3 & $32 h$ & 1 & $0.3309(5)$ & $0.2436(3)$ & $0.7497(17)$ & $0.0048(9)$ \\
\hline $\mathrm{Mn} 4$ & $32 h$ & 1 & $0.3372(4)$ & $0.3279(3)$ & $0.4968(14)$ & $0.0012(9)$ \\
\hline Mn5 & $32 h$ & 1 & $0.3304(4)$ & $0.5848(4)$ & $0.7491(18)$ & $0.0029(7)$ \\
\hline $\mathrm{O} 1$ & $32 h$ & 1 & $0.2558(3)$ & $0.2575(3)$ & $0.7553(7)$ & $0.0097(12)$ \\
\hline $\mathrm{O} 2$ & $32 h$ & 1 & $0.2557(2)$ & $0.3270(2)$ & $0.163(7)$ & $0.0016(7)$ \\
\hline $\mathrm{O} 3$ & $32 h$ & 1 & $0.2479(2)$ & $0.5897(2)$ & $0.7333(7)$ & $0.0047(9)$ \\
\hline $\mathrm{O} 4$ & $32 h$ & 1 & $0.3268(2)$ & $0.2456(2)$ & $0.5233(6)$ & $0.0015(7)$ \\
\hline O6 & $32 h$ & 1 & $0.3252(2)$ & $0.5868(2)$ & $0.5138(7)$ & $0.0047(7)$ \\
\hline $\mathrm{O} 7$ & $32 h$ & $0.788(13)$ & $0.3376(3)$ & $0.3332(3)$ & $0.2699(8)$ & $0.0056(9)$ \\
\hline O8 & $32 h$ & 1 & $0.3387(3)$ & $0.50966(17)$ & $0.7609(7)$ & $0.0024(7)$ \\
\hline O9 & $32 h$ & 1 & $0.3371(3)$ & $0.5841(3)$ & $0.9868(8)$ & $0.0127(11)$ \\
\hline
\end{tabular}

Space group $F d d d, a=2.47438(6) \mathrm{nm}, b=2.48352(6) \mathrm{nm}, c=0.819577(18) \mathrm{nm}, R_{\mathrm{wp}}=6.31, R_{\mathrm{p}}=4.64, S=R_{\mathrm{wp}} / R_{\mathrm{e}}=1.0499, R_{\mathrm{I}}=1.73, R_{F}=2.06$.

Table 4 Neutron Rietveld refinement results at $260 \mathrm{~K}$ for $\mathrm{LiMn}_{2} \mathrm{O}_{4-\delta}(\delta=0.132)$ (sample A).

\begin{tabular}{|c|c|c|c|c|c|c|}
\hline Atom & Site & $g$ & $x$ & $y$ & $z$ & $U / \mathrm{nm}^{2}$ \\
\hline Li1 & $32 h$ & 1 & $0.2896(14)$ & $0.2869(11)$ & $0.128(4)$ & $0.0075(8)$ \\
\hline Li2 & $16 e$ & 1 & $0.2934(17)$ & $5 / 8$ & $1 / 8$ & $=\operatorname{Li1}(U)$ \\
\hline $\mathrm{Li} 4$ & $16 f$ & 1 & $3 / 8$ & $0.5474(16)$ & $3 / 8$ & $=\operatorname{Li1}(U)$ \\
\hline Mn1 & $16 d$ & 1 & $1 / 4$ & $1 / 4$ & $1 / 2$ & $0.0016(13)$ \\
\hline $\operatorname{Mn} 2$ & $32 h$ & 1 & $0.2511(3)$ & $0.3337(5)$ & $0.7530(15)$ & $0.0018(8)$ \\
\hline Mn3 & $32 h$ & 1 & $0.3311(5)$ & $0.2435(3)$ & $0.7512(17)$ & $0.0048(9)$ \\
\hline $\mathrm{Mn} 4$ & $32 h$ & 1 & $0.3360(4)$ & $0.3274(3)$ & $0.4969(14)$ & $0.0012(9)$ \\
\hline Mn5 & $32 h$ & 1 & $0.3304(4)$ & $0.5848(4)$ & $0.7491(18)$ & $0.0029(7)$ \\
\hline $\mathrm{O} 1$ & $32 h$ & 1 & $0.2561(3)$ & $0.2585(3)$ & $0.7561(7)$ & $0.0097(12)$ \\
\hline $\mathrm{O} 2$ & $32 h$ & 1 & $0.2565(2)$ & $0.3279(2)$ & $0.5170(7)$ & $0.0016(7)$ \\
\hline $\mathrm{O} 3$ & $32 h$ & 1 & $0.2479(2)$ & $0.5897(2)$ & $0.7333(7)$ & $0.0047(9)$ \\
\hline $\mathrm{O} 4$ & $32 h$ & 1 & $0.3268(2)$ & $0.2453(2)$ & $0.5237(6)$ & $0.0067(9)$ \\
\hline O6 & $32 h$ & 1 & $0.3252(2)$ & $0.5871(2)$ & $0.5137(6)$ & $0.0059(9)$ \\
\hline $\mathrm{O} 7$ & $32 h$ & $0.790(13)$ & $0.3372(3)$ & $0.3335(3)$ & $0.2699(7)$ & $0.0035(9)$ \\
\hline O8 & $32 h$ & 1 & $0.3393(2)$ & $0.50965(17)$ & $0.7602(7)$ & $0.0030(8)$ \\
\hline O9 & $32 h$ & 1 & $0.3372(3)$ & $0.5845(2)$ & $0.9872(8)$ & $0.0117(11)$ \\
\hline
\end{tabular}

Space group $F d d d, a=2.47491(5) \mathrm{nm}, b=2.48514(6) \mathrm{nm}, c=0.819838(17) \mathrm{nm}, R_{\mathrm{wp}}=6.02, R_{\mathrm{p}}=4.42, S=R_{\mathrm{wp}} / R_{\mathrm{e}}=1.0476, R_{\mathrm{I}}=1.66, R_{F}=1.87$.

shows the neutron diffraction $(400)_{c}$ peaks at low temperatures. The samples A and B show the peak splittings at $270 \mathrm{~K}$, corresponding to the orthorhombic symmetry, and the sample $\mathrm{C}$ shows only peak broadening at $150 \mathrm{~K}$. The peak splittings at $150 \mathrm{~K}$ shown in Fig. 2(b) indicates that the orthorhombic distortion decreased with decreasing $\delta$ from 0.132 to 0.006

The peak splittings below $280 \mathrm{~K}$ for the sample A were indexed by an orthorhombic cell. There are also small diffraction peaks corresponding to the superlattice reflections. The electron diffraction measurements indicated the cubic symmetry with $a \sim 0.824 \mathrm{~nm}$ at $300 \mathrm{~K}$. At $120 \mathrm{~K}$, the superlattice reflections were observed and indexed by the $3 a \times 3 a \times a$ cell based on the parent cubic cell of $a$. The orthorhombic lattice of space group $F d d d^{9)}$ is used for our structure refinement. Figure 3 shows the temperature dependence of the lattice parameters of the sample A. With decreasing temperature from $300 \mathrm{~K}$, the orthorhombic distortion appeared at $290 \mathrm{~K}$ and the distortion decreased gradually with decreasing temperature. Around $40 \mathrm{~K}$, the $b$ axis increases, and the $a$ and $b$ axes become close to each other. This anomaly corresponds to the anti-ferromagnetic 


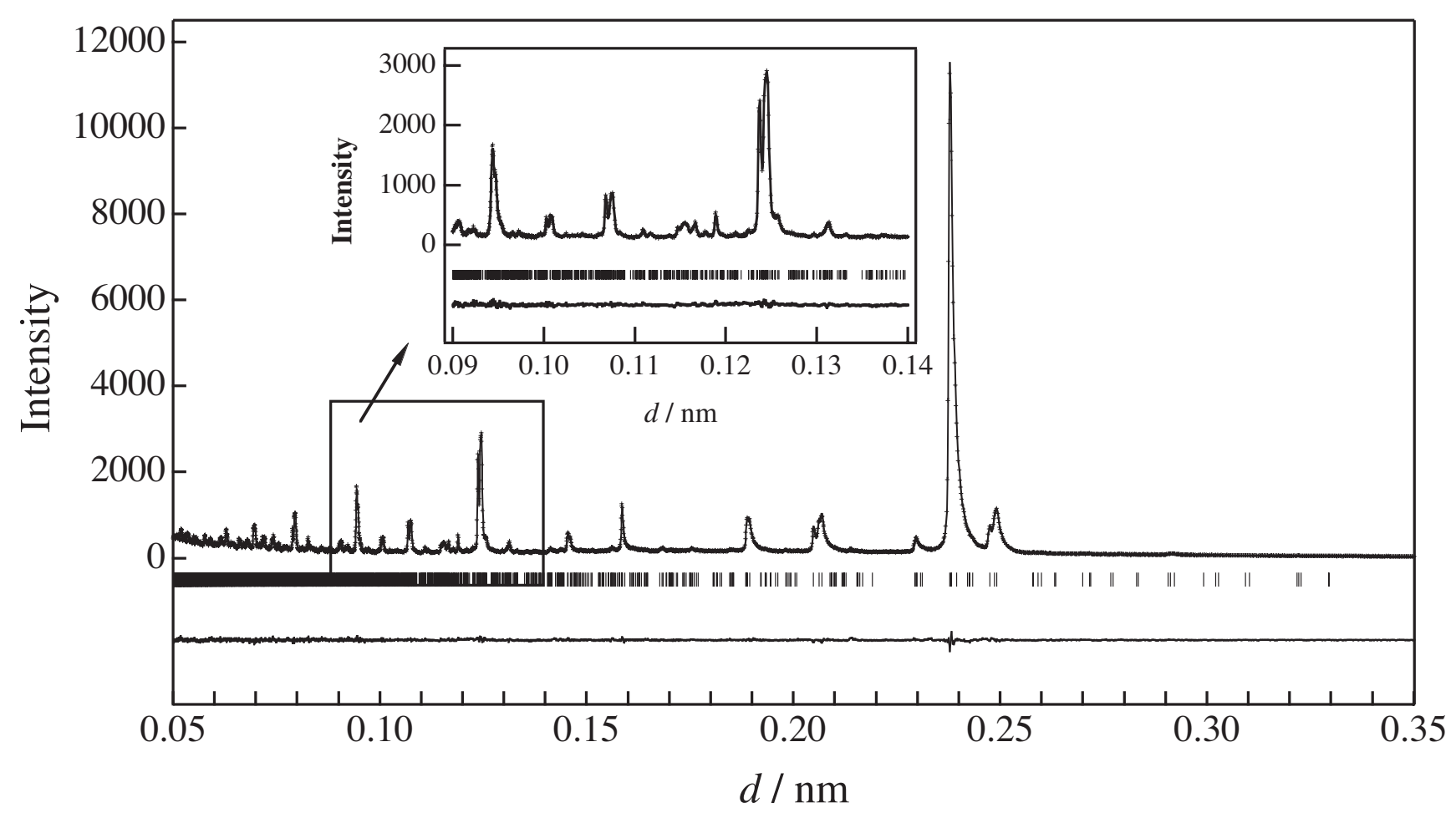

Fig. 5 Neutron Rietveld refinement pattern for the sample A at $150 \mathrm{~K}$.
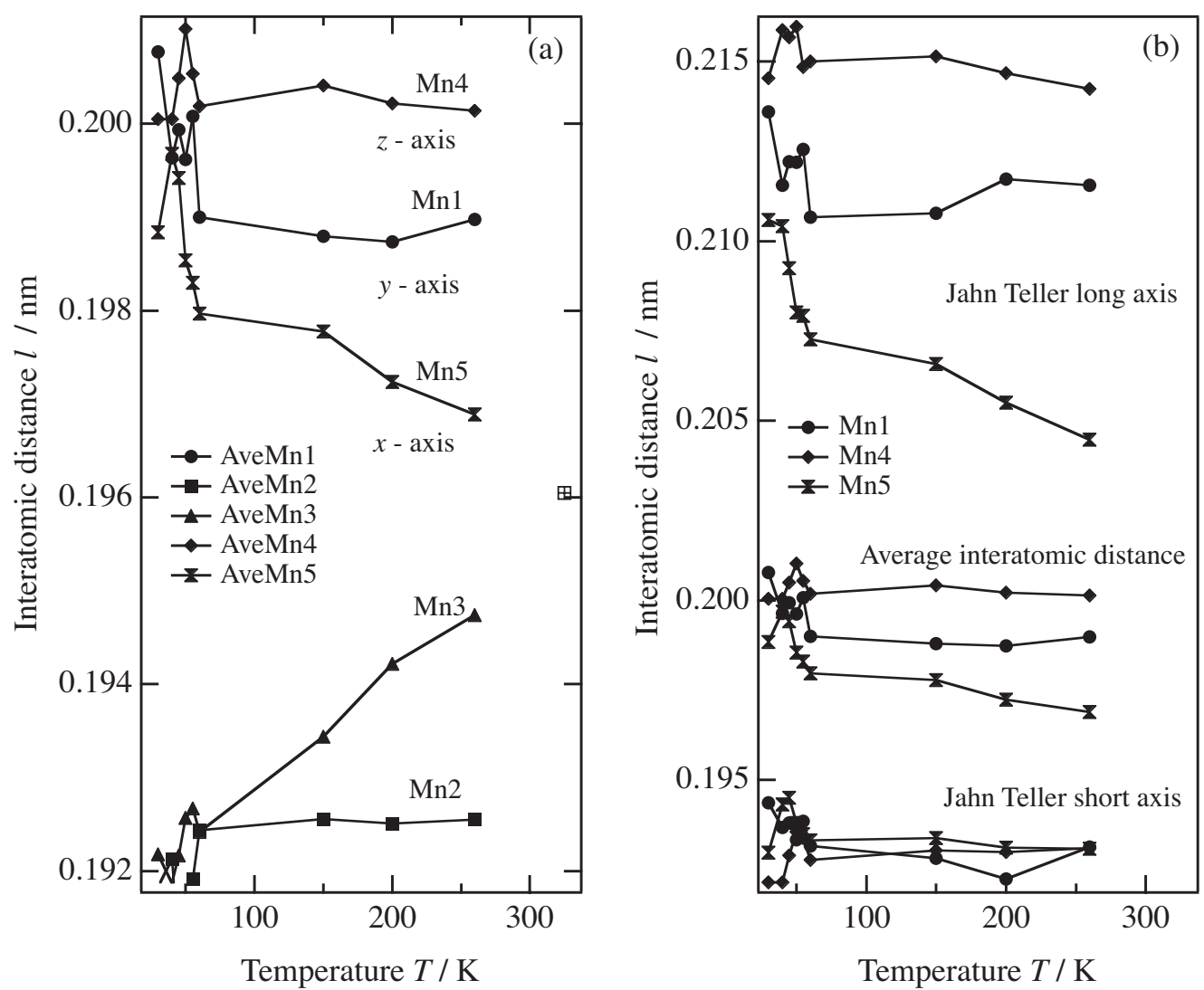

Fig. 6 Temperature dependence of the average Mn-O distances (a). Temperature dependence of the average distance of the Jahn-Teller long axes and short axes (b). 
transition, and the phase below $40 \mathrm{~K}$ is close to tetragonal as indicated previously. ${ }^{17)}$

For the sample $\mathrm{C}$, on the other hand, the peak splittings were not clearly observed at low-temperatures. However, peak-shift to higher angles was observed from 240 to $210 \mathrm{~K}$, which is the opposite direction of the shift from 290 to $240 \mathrm{~K}$. Peak broadening was also observed. This indicates the phase transition around $240 \mathrm{~K}$. The lattice parameter was then refined using the same symmetry as the sample A. Figure 4 shows temperature dependence of the lattice parameters for $\mathrm{LiMn}_{2} \mathrm{O}_{3.984}$ (sample C). DSC measurements on the sample $\mathrm{C}$ showed no heat anomaly corresponding to the phase transition around room temperature, while the samples A and B showed large heat anomaly due to the cubic-orthorhombic phase transition. Adiabatic calorimetry measurement on the sample $\mathrm{C}$, on the other hand, indicated small anomaly in the $C p$ vs. $T$ curves around $220 \mathrm{~K}$ corresponding to the charge ordering phase transition. ${ }^{19)}$ The lattice parameter changes indicates the distortion below $240 \mathrm{~K}$ which is consistent with the calorimetry results.

Previously, the lattice symmetry of the low-temperature phases was considerably confusing. This is caused by the difference in the compositions of the spinels used for the structure determination. The charge-ordering phase transition exists for all the samples examined in the present study, and the extent of orthorhombic distortion decreased with decreasing $\delta$. The tetragonal symmetry reported previously ${ }^{10)}$ corresponds to the phase with small distortion of the lowtemperature phase with large amount of vacancy. We did not used the two-phase mixture model with the tetragonal and cubic phases $^{11)}$ for the refinement of low-temperature structures because all the diffraction peaks were successfully indexed by the orthorhombic structure model.

The low-temperature structure of the sample A was determined using neutron diffraction data and the $3 a \times 3 a \times$ $a$ lattice with oxygen vacancy in the structure. The structure model was similar to that reported previously. ${ }^{9)}$ Tables 2-4 summarize the refinement results at 150,200 , and $260 \mathrm{~K}$. The final $R$ factors, lattice and structural parameters with their estimated standard deviations in parentheses are indicated. Figure 5 illustrates the profile fit and difference pattern at $150 \mathrm{~K}$; the calculated pattern fits the observed one fairly well. The Li site splits from 1 to 4 , the Mn ions site from 1 to 5 sites, and the $\mathrm{O}$ site from 1 to 9 at low temperatures. During the refinements, the $\mathrm{O} 7$ site was found to be different from other oxygen site. The occupation parameter was then refined. The refinement suggests the existence of oxygen vacancy at the $\mathrm{O} 7$ site.

Figure 6 shows the temperature dependence of the Mn-O bond distances. Two different sets of $\mathrm{Mn}-\mathrm{O}$ distances are observed, three of five $\mathrm{Mn}-\mathrm{O}$ distances are larger than the other set of two Mn-O distances. Longer distances of Mn4-O, $\mathrm{Mn1-O}$, and Mn5-O corresponds to lower valence state($\left.\mathrm{Mn}^{(3+)+\gamma}\right)$, and shorter distances of Mn3-O and Mn2-O to higher valence state $\left(\mathrm{Mn}^{(4+)-\gamma}\right)$. The elongated Jahn-Teller axis of the trivalent manganese ions, Mn1(16d), Mn4(32h) and $\mathrm{Mn} 5(32 h)$ ordered along the $z, x$, and $y$ direction, respectively. Particularly, the bond distance of the elongated axis in Mn5-O increased with decreasing temperature. This corresponds to the charge-ordering process accompanying

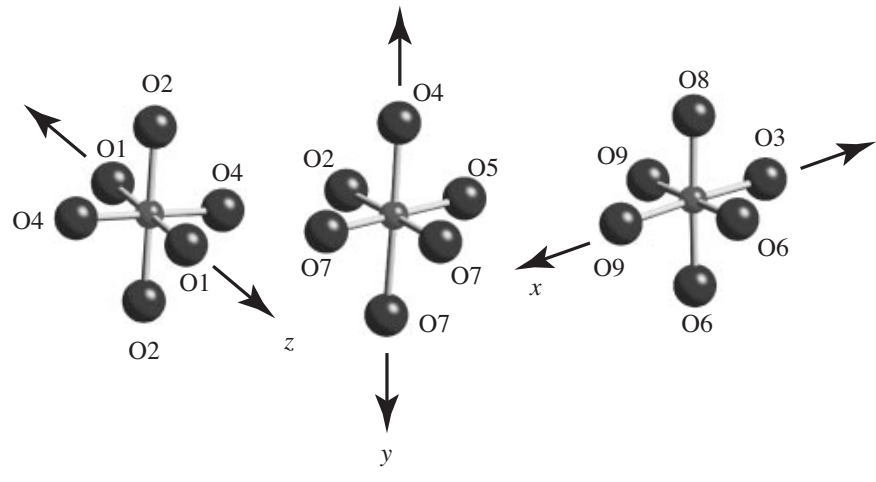

(a) Mn1

(b) Mn4

(c) Mn5

Fig. 7 Schematic drawing of the $\mathrm{MnO}_{6}$ octahedra of trivalent manganese ions. The elongated axes ordered in different directions for each Mn sites.

electron transfer from the Mn3 to Mn5 sites. Figure 7 shows schematic drawing of the $\mathrm{MnO}_{6}$ octahedra of trivalent manganese ions. The elongated Jahn-Teller long axis of the Mn1, Mn4, and Mn5 sites ordered along $z, y$, and $x$ directions, respectively. For the tetragonal spinel obtained by quenching from $920^{\circ} \mathrm{C}$, the elongated octahedral axis ordered only in $z$ direction, that caused the tetragonal symmetry. ${ }^{4)}$ The orthorhombic phase has different orientation of the elongated axis, and this gives the structure with the lattice symmetry of Fddd. The average Mn4-O distance is larger than those of Mn1-O and Mn5-O distances, indicating smaller $\gamma$ value in $\mathrm{Mn}^{(3+)+\gamma}$. The $\mathrm{O} 7$ site is closely situated to the Mn4 site.

The structure parameters of the samples B and C were refined using the neutron diffraction data at $150 \mathrm{~K}$ and the structure model similar to the sample A at $150 \mathrm{~K}$. The interatomic distances were calculated base on the refined structure parameters. Figure 8 shows the average Mn-O

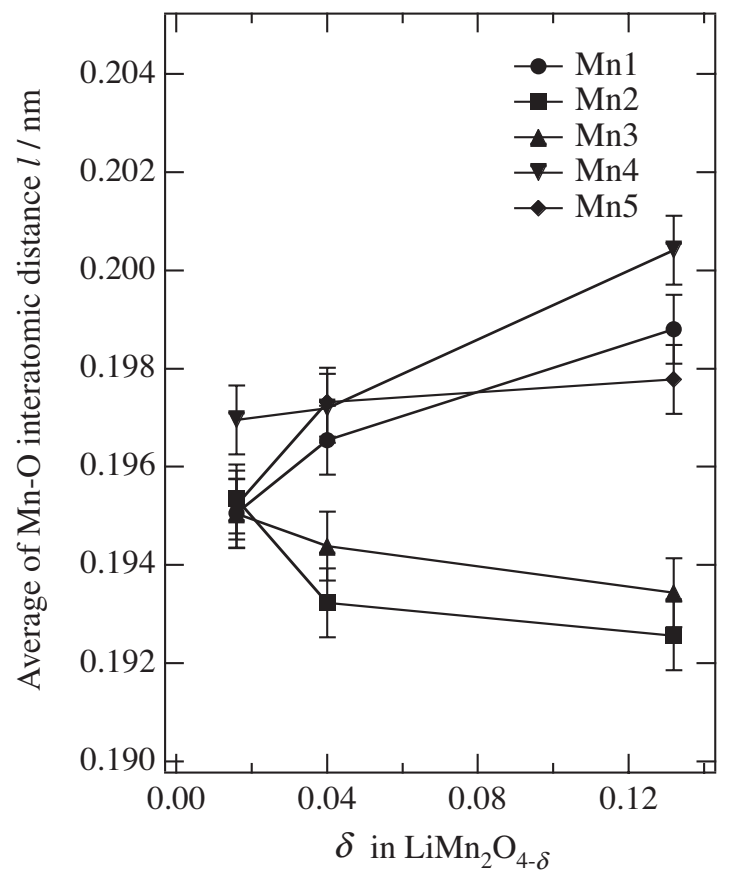

Fig. 8 Oxygen vacancy dependence of the average distance of the JahnTeller long-axes and short-axes at $150 \mathrm{~K}$. 
distances for five manganese sites at $150 \mathrm{~K}$ as a function of oxygen vacancy $\delta$. Longer Mn-O distances corresponds to the $\mathrm{Mn}^{(3+)+\gamma}$ state, and shorter Mn-O distances to the $\mathrm{Mn}^{(4+)-\gamma}$ state. With decreasing oxygen vacancy, the difference between the $\mathrm{Mn}-\mathrm{O}$ (long) and $\mathrm{Mn}-\mathrm{O}$ (short) distances become small. The charge-ordering in the spinel structure is closely correlated to the amount of oxygen vacancy.

Previously, we reported the room temperature structures of lithium manganese oxide spinels with different synthesis conditions. ${ }^{20)}$ The phase transitions in $\mathrm{LiMn}_{2} \mathrm{O}_{4-\delta}$ depend on the amount of oxygen vacancy: (i) the charge-ordering transition shift from 300 to $250 \mathrm{~K}$, (ii) the anti-ferromagnetic transition also shift from 60 to $40 \mathrm{~K}$, and (iii) the short-range magnetic ordering (spin-glass-like behavior) shifts from 20 to $40 \mathrm{~K}$ with decreasing $\delta$. We concluded that the stoichiometric spinel $\mathrm{LiMn}_{2} \mathrm{O}_{4}$ was difficult to synthesize and the ideal composition might show no charge-ordering phase transition. The present X-ray and neutron diffraction study also indicated that the extent of orthorhombic distortion is related to the amount of oxygen vacancy. The neutron diffraction study suggests the vacancy at the $\mathrm{O} 7$ site which situates close to the Mn4 site with the lowest charge among the five manganese sites. The transition behavior observed for the spinel is closely correlated to the existence of oxygen vacancy.

\section{Acknowledgement}

This work was supported partly by a Grant-in-Aid from The Ministry of Education, Culture and Sports, Science and Technology of Japan, and partly by Genesis Research Institute.

\section{REFERENCES}

1) M. M. Thackeray, W. I. F. David, P. G. Bruce and J. B. Goodenough: Mater. Res. Bull., 18 (1983) 461.

2) Y. Xia and M. Yoshio: J. Electrochem. Soc., 144 (1997) 4186.

3) A. Yamada, K. Miura, K. Hinokuma and M. Tanaka: J. Electrochem. Soc., 142 (1995) 2149.

4) R. Kanno, A. Kondo, M. Yonemura, R. Gover, Y. Kawamoto, M. Tabuchi and T. Kamiyama, F. Izumi, C. Masquelier, and G. Rousse: $J$. Power Sources, 81 (1999) 542

5) P. Strobel, F. L. Cras, L. Seguin, M. Anne and J. M. Tarascon: J. Solid State Chem., 135 (1998) 132.

6) A. Yamada and M. Tanaka: Mater. Res. Bull., 30 (1995) 715.

7) A. Yamada: J. Electrochem. Soc., 122 (1996) 160.

8) K. Oikawa, T. Kamiyama, F. Izumi, B. C. Chakoumakos, H. Ikuta, M. Wakihara, J. Li and Y. Matsui: Solid State Ionics, 109 (1998) 35.

9) G. Rousse, C. Masquelier, J. Rodriguez-Carajal and M. Hervieu: Eletrochem. Solid-State Lett., 2(1) (1996) 6.

10) A. S. Wills, N. P. Raju and J. E. Greedan: Chem. Mater., 11 (1999) 1510.

11) X. Q. Yang, X. Sun, M. Balasubramanian, J. McBreen, Y. Xia, T. Sakai and M. Yoshio: Electrochem. Solid-State Lett., 4 (2001) A117.

12) J. Sugiyama, T. Atsumi, T. Hioki, S. Noda and N. Kamegashira: J. Power Sources., 68 (1997) 641.

13) J. Sugiyama, T. Atsumi, A. Koiwai, T. Sasaki, T. Hioki, S. Noda and N. Kamegashira: J. Phys. Condens. Matter., 9 (1997) 1729.

14) Y. Chida, H. Wada and K. Shizuka: J. Power Sources, 81-82 (1999) 454.

15) M. Hosoya, H. Ikuta, T. Uchida and M. Wakihara: J. Electrochem. Soc., 144 (1997) L52.

16) F. Izumi, “The Rietveld Method,” ed. by R. A. Young: Oxford Univ. Press, Oxford, (1993) Chap. 13.

17) H. Hayakawa, T. Takada, H. Enoki and E. Akiba: Powder Diffraction, 15, (2000) 19.

18) G. Rousse, C. Masquelier, J. Rodriguez-Carvajal, E. Elkaim, J.-P. Lauriat and J. L. Martinez: Chem. Mater., 12 (1999) 3629.

19) M. Tachibana, T. Tojo, H. Kawaji, T. Atake, H. Yonemura and R. Kanno: Phys. Rev. B, 68 (2003) 094421.

20) M. Yonemura, A. Yamada, H. kobayashi, M. Tabuchi, T. Kamiyama, Y. Kawamoto, R. Kanno, in printing. 